\title{
Dual enteric and respiratory tropisms of winter dysentery bovine coronavirus in calves
}

\author{
S. J. Park ${ }^{1}$, G. Y. Kim ${ }^{2}$, H. E. Choy ${ }^{3}$, Y. J. Hong ${ }^{3}$, L. J. Saif ${ }^{4}$, J. H. Jeong ${ }^{1}$, S. I. Park ${ }^{1}$, \\ H. H. Kim ${ }^{1}$, S. K. Kim ${ }^{1}$, S. S. Shin ${ }^{1}$, M. I. Kang ${ }^{1}$, K. O. Cho ${ }^{1}$ \\ ${ }^{1}$ Biotherapy Human Resources Center, College of Veterinary Medicine, Chonnam National University, \\ Gwangju, South Korea \\ ${ }^{2}$ College of Health and Well Fare, Dongshin University, Naju, Chonnam, South Korea \\ ${ }^{3}$ Genome Research Center for Enteropathogenic Bacteria, Chonnam National University Medical College, \\ Gwangju, South Korea \\ ${ }^{4}$ Ohio Agricultural Research and Development Center, Food Animal Health Research Program, \\ Department of Veterinary Preventive Medicine, The Ohio State University, Wooster, Ohio, USA
}

Received 5 December 2006; Accepted 4 May 2007; Published online 14 June 2007

(C) Springer-Verlag 2007

\section{Summary}

Although winter dysentery (WD), which is caused by the bovine coronavirus $(\mathrm{BCoV})$ is characterized by the sudden onset of diarrhea in many adult cattle in a herd, the pathogenesis of the WD-BCoV is not completely understood. In this study, colostrumdeprived calves were experimentally infected with a Korean WD-BCoV strain and examined for viremia, enteric and nasal virus shedding as well as for viral antigen expression and virus-associated lesions in the small and large intestines and the upper and lower respiratory tract from 1 to 8 days after an oral infection. The WD-BCoV-inoculated calves showed gradual villous atrophy in the small intestine and a gradual increase in the crypt depth of the large intestine. The WD-BCoV-infected animals showed epithelial damage in nasal turbinates, trachea and

Correspondence: K. O. Cho, Biotherapy Human Resources Center, College of Veterinary Medicine, Chonnam National University, Gwangju 500-757, South Korea e-mail: choko@chonnam.ac.kr lungs, and interstitial pneumonia. The WD-BCoV antigen was detected in the epithelium of the small and large intestines, nasal turbinates, trachea and lungs. WD-BCoV RNA was detected in the serum from post-inoculation day 3. These results show that the WD-BCoV has dual tropism and induces pathological changes in both the digestive and respiratory tracts of calves. To our knowledge, this is the first detailed report of dual enteric and respiratory tropisms of WD-BCoV in calves. Comprehensive studies of the dual tissue pathogenesis of the $\mathrm{BCoV}$ might contribute to an increased understanding of similar pneumoenteric $\mathrm{CoV}$ infections in humans.

\section{Introduction}

The coronaviruses $(\mathrm{CoV})$ constitute a genus in the family Coronaviridae, large enveloped viruses ranging in diameter from 100 to $120 \mathrm{~nm}$, that possess a single-stranded, positive-sense RNA genome of $27-32 \mathrm{~kb}$ in length and cause important diseases in animals [23]. Coronaviruses (CoVs) can be divided 
into three distinct groups according to their natural hosts, nucleotide sequences, and serologic relationships [23]. The bovine $\mathrm{CoV}$ (BCoV), which belongs to the $\mathrm{CoV}$ group 2a, contains five major structural proteins: the nucleocapsid $(\mathrm{N})$, the transmembrane, the spike $(\mathrm{S})$, the small membrane and the hemagglutinin/esterase (HE) proteins, the latter being unique to some group $2 \mathrm{CoVs}$ [23]. Although both $\mathrm{S}$ and $\mathrm{HE}$ glycoproteins of the $\mathrm{BCoV}$ use $\mathrm{N}$ acetyl-9-O acetyl neuraminic acid as a receptor determinant to initiate an infection, the S glycoprotein requires a smaller amount of these receptors on the surface of erythrocytes for agglutination than the HE protein $[32,33]$. Therefore, the S glycoprotein is the major hemagglutinin of BCoV [32] and is believed to be responsible for the primary attachment of $\mathrm{BCoV}$ to other cell surface receptors [33]. Consequently, the variation in the host range and tissue tropism of CoVs may largely be due to variations in the $\mathrm{S}$ gycoprotein [15].

Bovine coronaviruses cause three different clinical diseases: severe calf diarrhea (CD), winter dysentery (WD) in adult cattle, and respiratory tract infections in calves and feedlot cattle [7, 24, 25, $31]$. In addition, the virus has been associated with a new proposed clinical form, warmer season dysentery in adult cattle [27]. Because the porcine respiratory $\mathrm{CoV}$ emerged from porcine transmissible gastroenteritis CoV (TGEV) through deletion mutations with reduced enteropathogenicity and a loss of hemagglutinating activity [29] but pronounced tropism for lung tissue, many attempts have been made to isolate and identify a potential deletion mutant of $\mathrm{BCoV}$ with altered tissue tropism or virulence. Despite their involvement in the different clinical forms, only minor variations in the $S$ and $\mathrm{HE}$ genes among the strains associated with $\mathrm{CD}$, $\mathrm{WD}$, and bovine respiratory $\mathrm{CoV}(\mathrm{BRCoV})$ have been reported [9]. Moreover, only one serotype has been identified in the $\mathrm{BCoV}$ isolates or strains, even though the data obtained from serological studies, using monoclonal and polyclonal antibodies, suggested the existence of serologically different subtypes of $\mathrm{BCoV}$ [40]. Animals that have recovered from an infection with the $\mathrm{BRCoV}, \mathrm{CD}$, or WD strains were protected from $\mathrm{BCoV}$-associated diarrhea after a challenge exposure to either a hetero- logous or homologous strain of the $\mathrm{BCoV}$ [6], which suggests that only one BCoV serotype exists [40].

Winter dysentery is characterized by a sudden onset of often bloody diarrhea that spreads rapidly in adult cattle in a herd and has been reported in many parts of the world [30]. The WD-affected cattle lose body condition, resulting in a dramatic decrease in milk production, and many animals show signs of respiratory disease (nasal discharge) [30]. Recently, WD was successfully reproduced experimentally in BCoV-seronegative adult cattle and calves [39]. However, the digestive and respiratory pathogenesis of WD-BCoV in calves is so far unknown. This study attempted to answer the following three questions: 1) where in the digestive tract of calves does WD-BCoV replicate and cause pathological changes? 2) does WD-BCoV replicate and cause damage to the respiratory tract of calves? 3 ) if so, in which cells of the respiratory tract does WD$\mathrm{BCoV}$ replicate and cause damage? Accordingly, the digestive tract (small and large intestine) and the respiratory tracts including the nasal turbinates, trachea and lungs, were sampled from colostrumdeprived (Cols-D) calves inoculated with a Korean WD strain, KWD3, and analyzed for $\mathrm{BCoV}$ antigens by histopathology and immunohistochemistry, as well as by a scanning electron microscopy (SEM). In addition, the level of fecal and nasal virus shedding (detected by RT-PCR, nested PCR and ELISA) was assessed. The results of this study suggest that WD$\mathrm{BCoV}$ has dual tropism for the digestive and respiratory tracts of calves and induces pathological changes in these tissues by destroying the epithelium.

\section{Materials and methods}

\section{WD-BCoV inoculum}

The KWD3 strain of WD-BCoV was originally isolated and identified from a fecal sample from an adult cow with WD $[18,19]$. The KWD3 strain was passaged 6 times in human rectal adenocarcinoma cells (HRT-18G; ATCC). The virus titer of the KWD3 strain was determined using a cell culture immunofluorescence (CCIF) assay with fluorescein-isothiocyanate-conjugated hyperimmune antisera to the Mebus strain of the $\mathrm{BCoV}$, using a slight modification of a method described previously [41].

The mock-infected HRT-18G tissue culture supernatant was used for the mock inoculation of a control Cols-D calf 


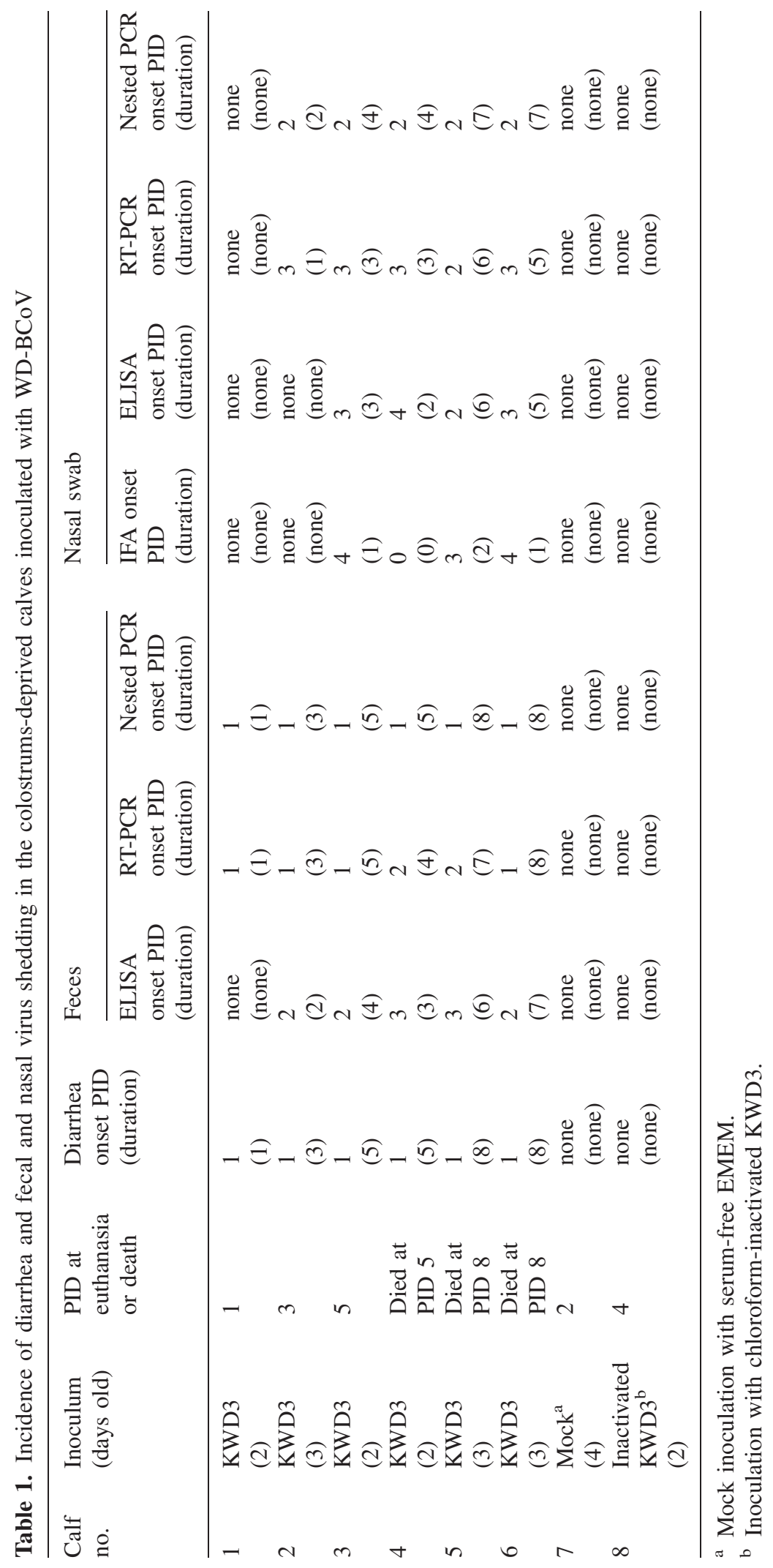


through the oral route. The possibility that the RT-PCR or nested PCR can detect the residual BCoV inoculum after inoculation was tested by inoculating another Cols-D calf with the inactivated KWD3 strain. The KWD3 strain was inactivated by chloroform treatment, as described previously [6].

\section{Animals and experimental design}

A total of eight 2- to 4-day-old Cols-D Holstein calves were used in this study. They were fed commercial calf formula. Six calves were inoculated orally with $40 \mathrm{ml}$ of the tissue culture supernatant from the KWD3 strain containing a virus titer of $1.5 \times 10^{8}$ immunofluorescence-forming units $/ \mathrm{ml}$ by the CCIF assay (Table 1). One calf was inoculated orally with $40 \mathrm{ml}$ of the mock-infected HRT-18G tissue culture supernatant, and the remaining calf was inoculated with $40 \mathrm{ml}$ of the inactivated KWD3 strain (Table 1).

After inoculation, the rectal temperatures, respiratory signs, and color and consistency of the feces from each calf were evaluated daily. The consistency of the feces was scored on a scale of $0-4$, with 0 representing firm; 1 , pasty; 2, semimucoid; 3, liquid and 4, profuse diarrhea. Nasal swabs and fecal samples were collected daily before and after inoculation, as described previously [13]. The inoculated calves were euthanized at post-inoculation day (PID) 1 (one calf), 3 (one calf), and 5 (one calf). One calf died at PID 5 and two calves died at PID 8. Blood samples were collected twice from each calf, prior to inoculation and immediately before euthanasia. Blood samples were collected from the heart and aorta of the three dead calves. The calves inoculated with the mock- and inactivated-KWD3 strain were euthanized at PID 2 and 4, respectively.

A necropsy of all the calves was performed immediately after death. At the necropsy, the intestinal tracts were removed and opened, and the small and large intestinal contents were collected. Samples from the intestinal segments were collected and immersed immediately in fixatives for the histological (10\% buffered formalin) and SEM (see below) examinations. The intestinal segments examined included the duodenum (approximately $15 \mathrm{~cm}$ caudal to the pyloric valve), jejunum (mid region of the small intestine), ileum (approximately $15 \mathrm{~cm}$ cranial to the ileocecal junction), cecum, colon, and rectum. Samples from the nasal turbinates, trachea, lungs, liver, spleen, heart, brain and kidneys were also collected and placed immediately in $10 \%$ buffered formalin or SEM fixatives (see below).

\section{Histological examination}

The formalin-fixed, paraffin-embedded tissue sections from each organ and tissue were stained with Mayer's hematoxylin and eosin and examined microscopically. The histological evaluation was performed in a blind manner on coded samples, and a comparison was made with the tissues from the mock-infected control.
Atrophy of the small intestinal villi and large intestinal glands was measured from histological sections of the duodenum, jejunum, ileum, cecum, colon and rectum. The small intestinal changes were scored according to the average villi/ crypt $(\mathrm{V} / \mathrm{C})$ ratio plus the grade of epithelial cell desquamation, which was measured as follows: $\mathrm{V} / \mathrm{C}$ ratio, $0=$ normal $(\mathrm{V} / \mathrm{C} \geqq 6: 1), 1=$ mild $(\mathrm{V} / \mathrm{C}=5.0$ to $5.9: 1), 2=$ moderate $(\mathrm{V} / \mathrm{C}=4.0$ to $4.9: 1), 3=$ marked $(\mathrm{V} / \mathrm{C}=3.0$ to $3.9: 1)$, $4=$ severe $(\mathrm{V} / \mathrm{C} \leqq 3.0: 1)$ and desquamation grade, $0=$ normal (no desquamation), $1=$ mild (cuboidal attenuation of tip villous epithelium), $2=$ moderate (desquamation of upper villous epithelium), $3=$ marked (desquamation of lower villous epithelium), $4=$ severe (desquamation of crypt epithelium). The large intestinal lesions were classified according to the grade of epithelial cell desquamation plus the grade of large intestinal gland atrophy as follows: desquamation grade, $0=$ normal (no desquamation), $1=$ mild (cuboidal attenuation of tip epithelium), $2=$ moderate (desquamation of tip epithelium), $3=$ marked (desquamation of upper middle epithelium), $4=$ severe (desquamation of lower middle epithelium). These changes were evaluated by measuring 10 randomly selected villi and large intestinal glands on the intestinal histological sections. This methodology is similar to that reported elsewhere [14].

Lesions in the nasal turbinates and trachea were classified according to the degree of degeneration (cell swelling and loss of cilia) and necrosis (characterized by pyknosis, karyorrhexis, karyolysis and the absence of nuclei associated with cell swelling and loss of cilia) of the epithelium as follows: $0=$ normal, $1=$ one to two degenerating cells, $2=$ three to five degenerating and necrotic cells, $3=$ many degenerating and necrotic cells. The lung lesions were scored according to the extent of interstitial pneumonia caused by the infiltration of macrophages and lymphoid cells in the interstitium, the accumulation of hyperplastic type II pneumonocytes, and the degree of the degeneration, necrosis and desquamation of the alveolar epithelial cells. The lesions were classified as follows: $0=$ normal, $1=$ scattered mild thickening of interstitium, and degeneration and necrosis of two or three, and desquamation of four to six alveolar epithelial cells, $2=$ multifocal moderate thickening of interstitium, and degeneration and necrosis of four to six, and desquamation of many alveolar epithelial cells, $3=$ diffuse severe thickening of the interstitium as well as degeneration, necrosis and desquamation of many alveolar epithelial cells.

\section{Detection of BCoV antigens in tissues by immunohistochemistry}

The distribution of the $\mathrm{BCoV}$ antigens in the tissues was evaluated through an immunohistochemical examination of the paraffin-embedded sections of each segment of the small and large intestines, nasal turbinates, trachea, and lungs, using a slight modification of the methodology described previously [8]. Three monoclonal antibodies [Mabs; BC 
$28 \mathrm{H} 1.2 \mathrm{C}$ against the $\mathrm{N}$ protein $(0.18 \mathrm{mg} / \mathrm{ml}), \mathrm{BC} 29 \mathrm{G} 7.2$ $\mathrm{C}$ against the $\mathrm{S}$ protein $(0.32 \mathrm{mg} / \mathrm{ml})$, and $\mathrm{BC} 22 \mathrm{~F} 8.3 \mathrm{C}$ against the $\mathrm{HE}$ protein $(0.2 \mathrm{mg} / \mathrm{ml})]$ of the DB2 strain of $\mathrm{BCoV}$, which were originally used for ELISA and Western blotting [36], were used as the primary Mabs. These Mabs were diluted 1:200 with phosphate-buffered saline, $\mathrm{pH} 7.4$, and applied to each section.

The antigen distribution in the small and large intestines was evaluated based on the number of antigen-positive cells in the villi and large intestinal glands and was measured as follows: $0=$ no positive cells, $1=$ one to two positive epithelial cells in the tip or upper part of the villi and large intestinal glands, $2=$ three to five positive epithelial cells scattered in the tip or upper part of the villi and large intestinal glands, $3=$ many positive epithelial cells in the tip or upper part of the villi and large intestinal glands, $4=$ positive in almost all epithelial cells in the tip and upper part of the villi and large intestinal glands. The antigen distribution in the nasal turbinates, trachea and lungs was examined using a score based on the number of antigen-positive cells, and was measured as follows: $0=$ no antigen-positive cells, $1=$ two to three antigen-positive epithelial cells scattered in the epithelium of the nasal turbinates, trachea, and in the epithelium of the alveoli, bronchioles and bronchus of the lungs, $2=$ four to six antigen-positive epithelial cells scattered in the epithelium of nasal turbinates, trachea, and in the epithelium of the alveoli, bronchioles and bronchus of the lungs, and $3=$ many antigenpositive epithelial cells scattered in the epithelium of the nasal turbinates, trachea, and in the epithelium of alveoli, bronchioles and bronchus of lungs. The number of antigen-positive cells in the organs or tissues was determined by light microscopy in 10 fields per section at a magnification of $400 \times$.

\section{Scanning electron microscopy}

Segments of the duodenum, jejunum, ileum, colon, nasal turbinates and trachea were fixed in a mixture containing $3 \%$ glutaraldehyde, $2 \%$ paraformaldehyde and $1.5 \%$ acrolein in $0.1 \mathrm{M}$ collidine buffer, $\mathrm{pH} 7.3$, as described previously [14]. The specimens were dehydrated in an ethanol-dry ice series and gently vacuum dried. The dried tissues were sputter coated with approximately $150 \AA$ of platinum, and were examined using a scanning electron microscope (JEOL, Tokyo, Japan).

\section{Detection of WD-BCoV in fecal, nasal swab and serum specimens}

1) Detection of $\mathrm{BCoV}$ antigen by ELISA. Indirect antigencapture ELISA using the anti-BCoV Mabs employed for immunohistochemistry (see above) as the capture antibodies was used to detect the $\mathrm{BCoV}$ in the nasal swab fluids and fecal suspensions, as described elsewhere [36].

2) Detection of WD-BCoV by IFA. The nasal epithelial cells were collected from the nasal swabs and processed for direct IF staining, as described elsewhere [35].
3) RT-PCR and nested PCR. RNA was extracted and purified from the fecal, nasal swab and serum specimens of the inoculated Cols-D calves using the techniques described elsewhere [6]. RT-PCR and nested PCR assays with the primer pairs specific for the $\mathrm{BCoV} N$ gene were performed as described elsewhere [6].

\section{Results}

\section{Diarrhea and virus shedding in Cols-D calves}

The KWD3 strain caused severe diarrhea in all the inoculated calves by PID 1, which persisted until euthanasia or the death of the animal (Table 1). Diarrhea was not observed in the inactivated KWD3-inoculated or the mock-inoculated calves. The calves inoculated with the KWD3 strain showed elevated body temperatures but the calves inoculated with the inactivated KWD3 or mock inoculum did not. None of the calves inoculated with the KWD3 strain showed any respiratory signs.

Table 1 shows the data of fecal and nasal virus shedding in Cols-D calves inoculated with the KWD3-BCoV. ELISA detected the BCoV antigen in the fecal samples of the inoculated calves on PID 3-8. BCoV RNA was similarly detected by RTPCR and nested PCR in the fecal samples on PID $1-8$. IFA detected the nasal shedding of the $\mathrm{BCoV}$ in 3 out of 6 virus-inoculated calves at PID 3-4 for 1 or 2 days. ELISA revealed nasal shedding of the $\mathrm{BCoV}$ in 4 out of 6 virus-inoculated calves at PID 2-3 for 2-6 days. RT-PCR revealed nasal shedding of the $\mathrm{BCoV}$ in 5 out of 6 virus-inoculated calves at PID 3 for 1-6 days. Nested PCR detected nasal shedding of the $\mathrm{BCoV}$ in 5 out of 6 virus-inoculated calves at PID 2 for 2-7 days. Fecal and nasal virus shedding was not detected in the inactivated $\mathrm{BCoV}$ and mock-inoculated calves by the above-described methods.

\section{Histological findings}

(i) Intestinal tract. In mock- or inactivated-BCoVinoculated calves, no changes were observed. The small intestines exhibited long and slender villi with short crypts (Fig. 1A and B). The large intestines contained intestinal glands, which 

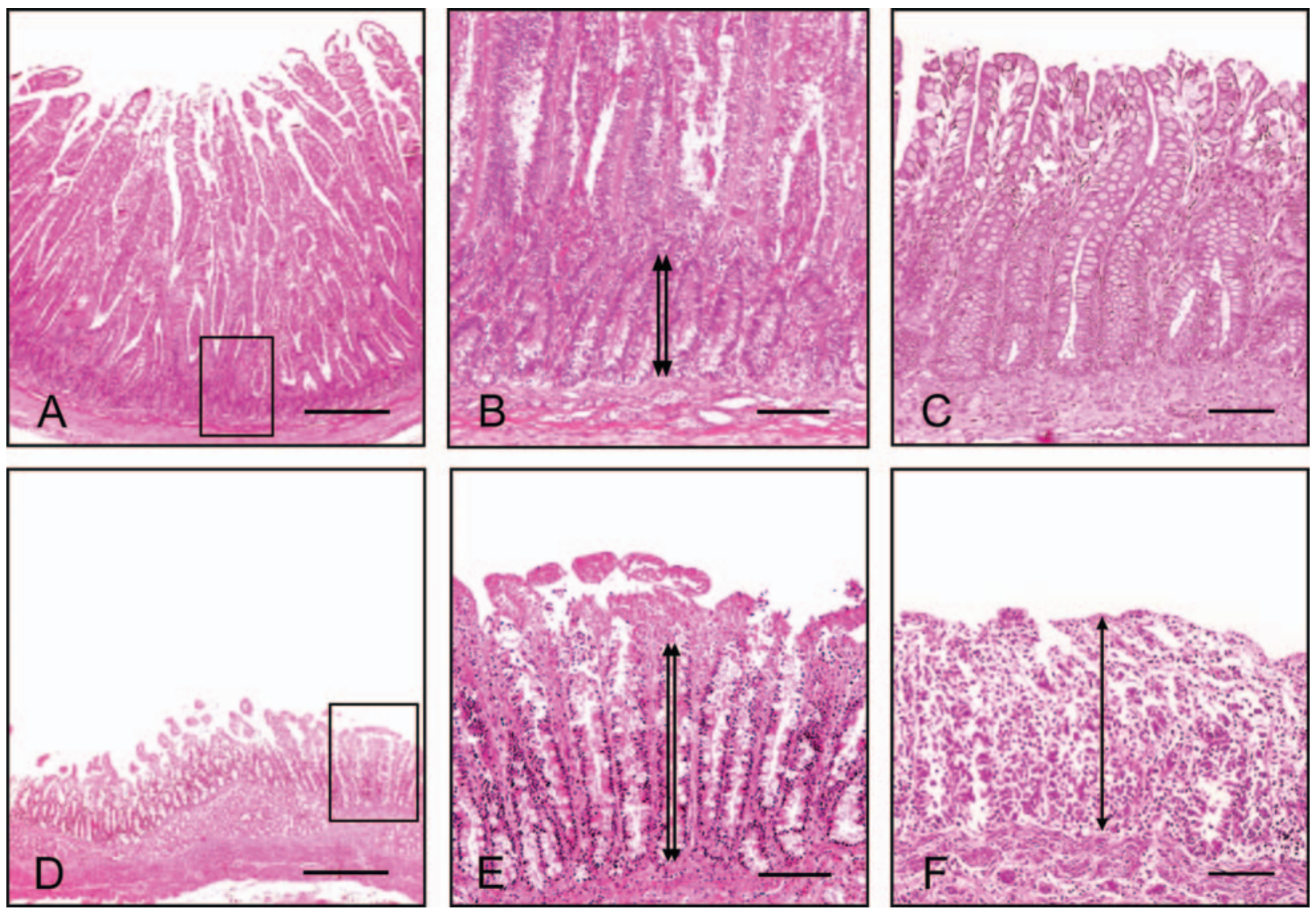

Fig. 1. Duodenum and colon; calf. A-C, control animal. A Unaltered duodenum showing long and slender villi. B High magnification of $\mathbf{A}$. Note the short normal crypts (double arrow). C Unaltered colon showing long and straight intestinal glands. D-F, BCoV-infected calf. D Severe villous atrophy in the duodenum at PID 8. E High magnification of D. Note the severe villous atrophy and marked hyperplasia of crypts (double arrow). F Moderate atrophy of the intestinal glands (arrow) with severe epithelial desquamation in the colon sampled at PID 8. Hematoxylin and eosin stain. Bar: A and D, $500 \mu \mathrm{m} ; \mathbf{B}$, $\mathbf{C}, \mathbf{E}$ and $\mathbf{F}, 200 \mu \mathrm{m}$

were elongated, straight and open to the luminal surface (Fig. 1C). In contrast, the BCoVinoculated calves showed mild to severe villous atrophy, widespread villous fusion and increased crypt depth in the small intestine (Fig. 1D and E), and moderate atrophy of the intestinal glands with severe epithelial desquamation in the large intestine (Fig. 1F) in the multiple cross sections.

Table 2 summarizes the histological changes in the small and large intestines from the virusinoculated calves. At PID 1, mild villous atrophy caused by necrosis and the desquamation of villous epithelial cells was confined in the duodenum. With time, the duodenal lesions became increasingly severe, and other parts of the intestine showed milder epithelial changes. Eventually, the duodenum showed severe villous atrophy and the other parts of the intestines including the jejunum, ileum, cecum, colon and rectum had moderate to marked mucosal lesions at PID 8 (Fig. 1D-F).

(ii) Respiratory tract. The calves inoculated with mock-inactivated $\mathrm{BCoV}$ did not exhibit any changes. They had pseudostratified ciliated columnar epithelium with goblet cells in the nasal turbinates, trachea and bronchus (Fig. 2A). The large bronchioles were lined with ciliated 


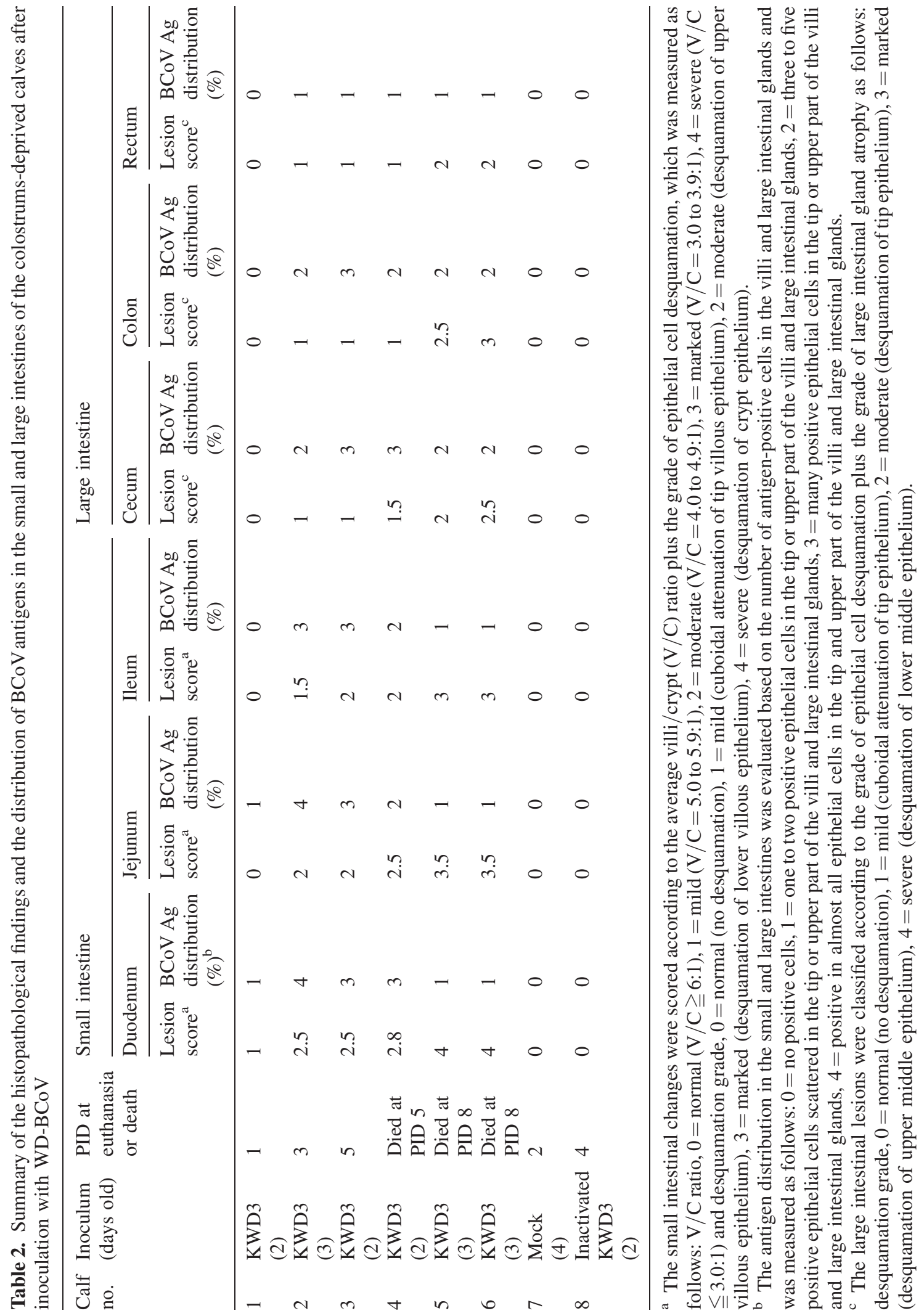



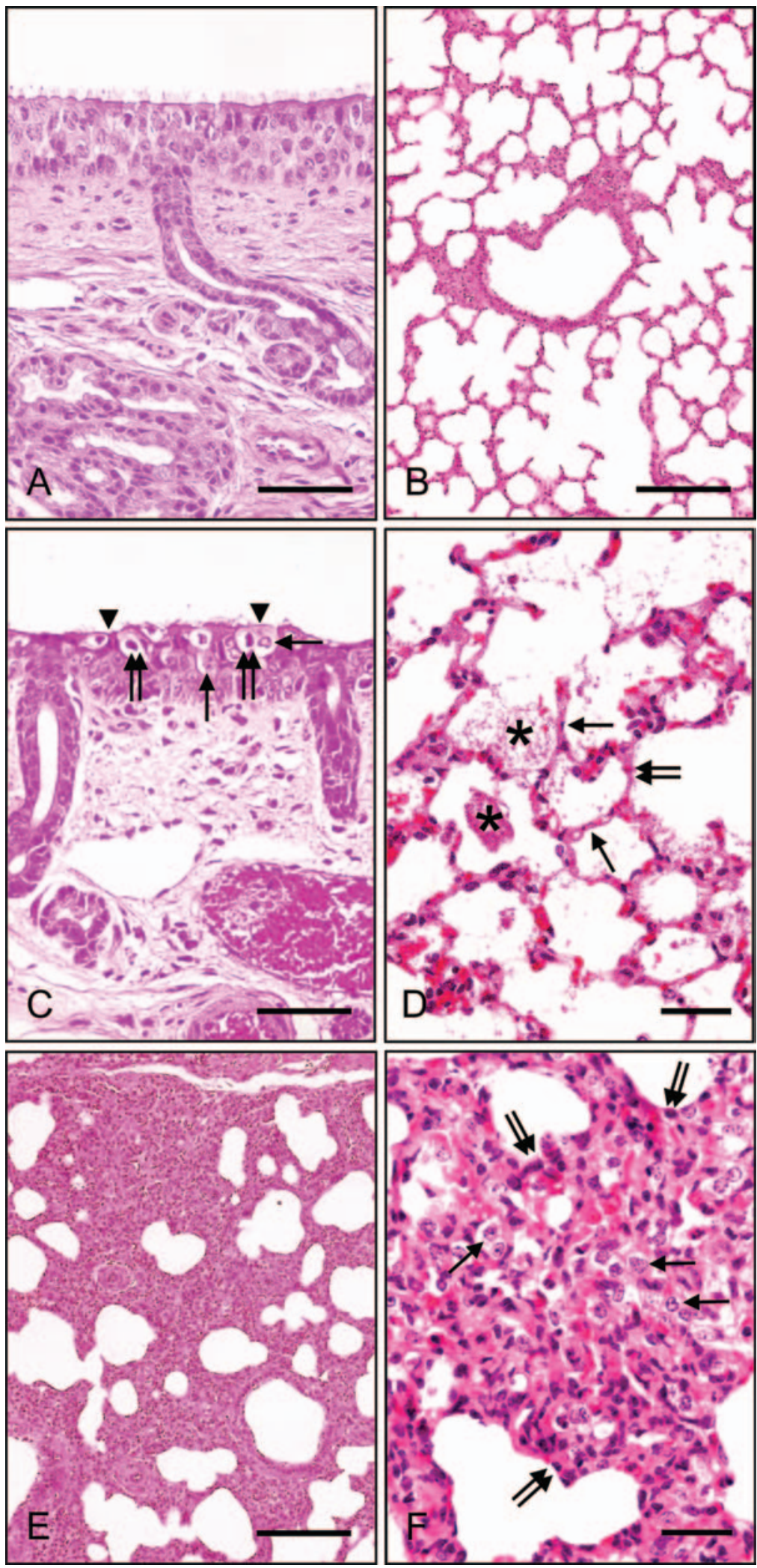

Fig. 2. Nasal turbinates and lungs; calf. A, B, control animal. A Unaltered nasal turbinates lined with pseudostratified ciliated columnar epithelium. B Unaltered lungs showing a thin alveoli wall. C-F, BCoV-infected calf. C Degeneration (arrows) and necrosis (double arrows) with cilia exfoliation (arrowheads) observed in the epithelium of nasal turbinates at PID 5. D Necrosis of alveolar lining cells (arrows) and denudation of the basal lamina (double arrow) with an exudation of fibrin (asterisks) observed in the lungs at PID 3. E Markedly expanded alveolar septa in the lungs at PID 5. F Higher magnification of $\mathbf{E}$. Note the intense infiltration of macrophages (arrows) with a few lymphoid cells in the alveolar septa and a lower numbers of hyperplastic type II pneumocytes (double arrows) in the alveolar wall. Hematoxylin and eosin stain. Bar: A, C, D and $\mathbf{F}, 50 \mu \mathrm{m}$; $\mathbf{B}$ and $\mathbf{E}, 400 \mu \mathrm{m}$ 


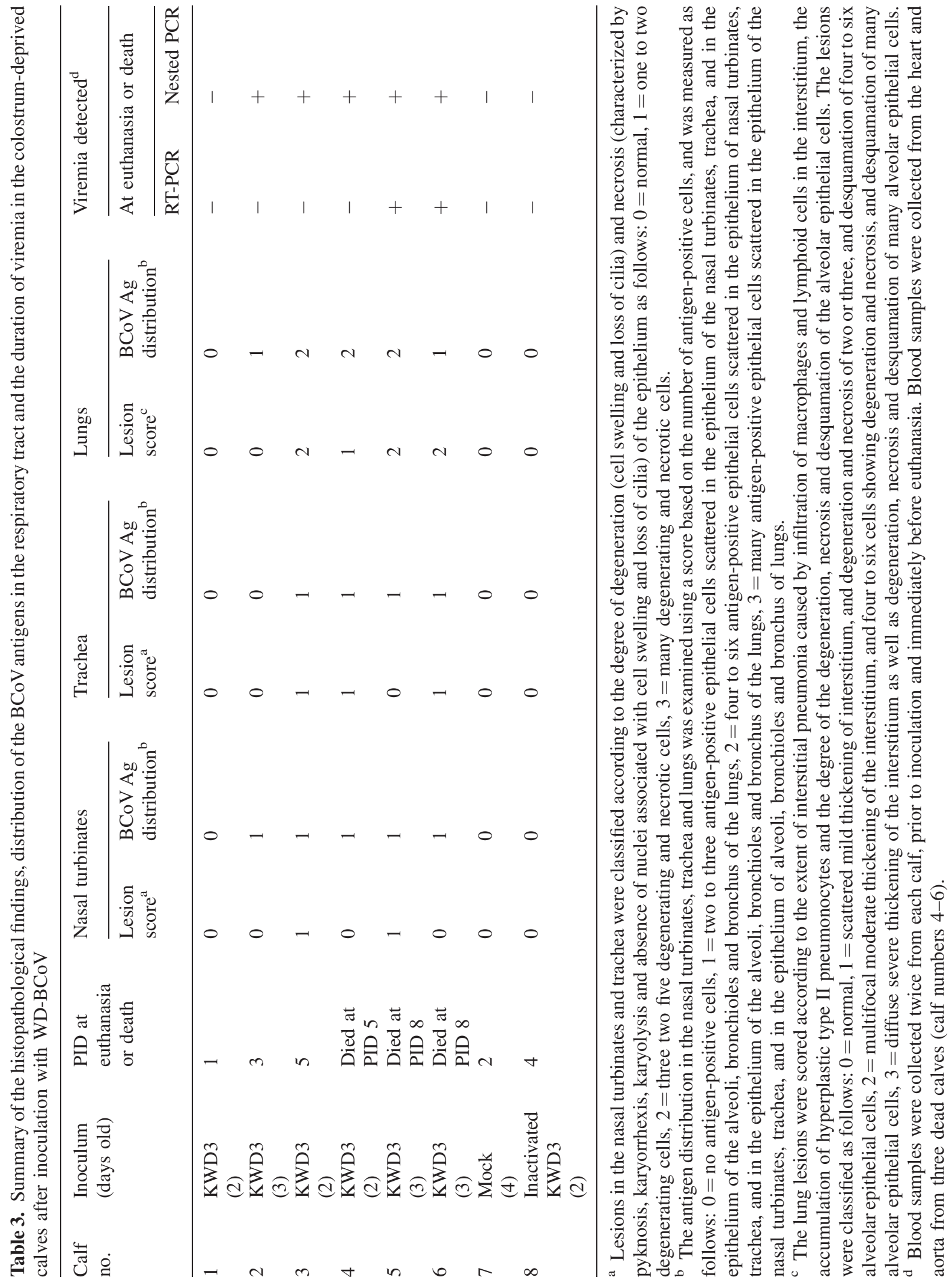


columnar cells, whereas the smallest (terminal) bronchioles were lined by ciliated cuboidal cells and, distally, with noncilated cells. The respiratory bronchioles were lined by a cuboidal epithelium, which became flattened distally. The alveoli were lined by flat alveolar epithelial cells (Fig. 2B). The changes in the nasal turbinates and trachea of the virus-inoculated calves were represented by multifocal epithelial cell degeneration (cell swelling and loss of cilia) and necrosis of a few epithelial cells (Table 3). At PID 1, no lesions were observed in the upper respiratory tract. However, at PID 3, few epithelial cells showing swelling with patch loss of cilia in the nasal turbinates and trachea were evident. Epithelial degeneration and occasional necrosis was detected in multifocal areas from PID 5 to 8 (Fig. 2C). A few lymphoid cells infiltrated the submucosa in some of the virus-inoculated calves.
The lungs from virus-infected calves showed no changes at PID 1 (Table 3). At PID 3, necrosis and sloughing of the alveolar lining cells was observed, resulting in the denudation of basal lamina (Fig. 2D). There was patch hyperplasia of type II pneumocytes and mild interstitial infiltration of macrophages. The alveolar sacs contained some foamy macrophages and desquamated alveolar lining cells, as well as some fibrin clots and eosinophilic proteinaceous fluid. At PID 5 and 8, there were an intense infiltration of macrophages with a few lymphoid cells in the alveolar septa and lower numbers of hyperplastic type II pneumocytes in the alveolar wall, leading to interstitial pneumonia (Fig. 2E and F). The alveolar cavities had a few foamy macrophages and desquamated alveolar lining cells, as well as some fibrin and eosinophilic proteinaceous fluid. The epithelia of the alveoli, bronchi and bronchioles were often desquamated or necrotic. These changes are listed in Table 3.
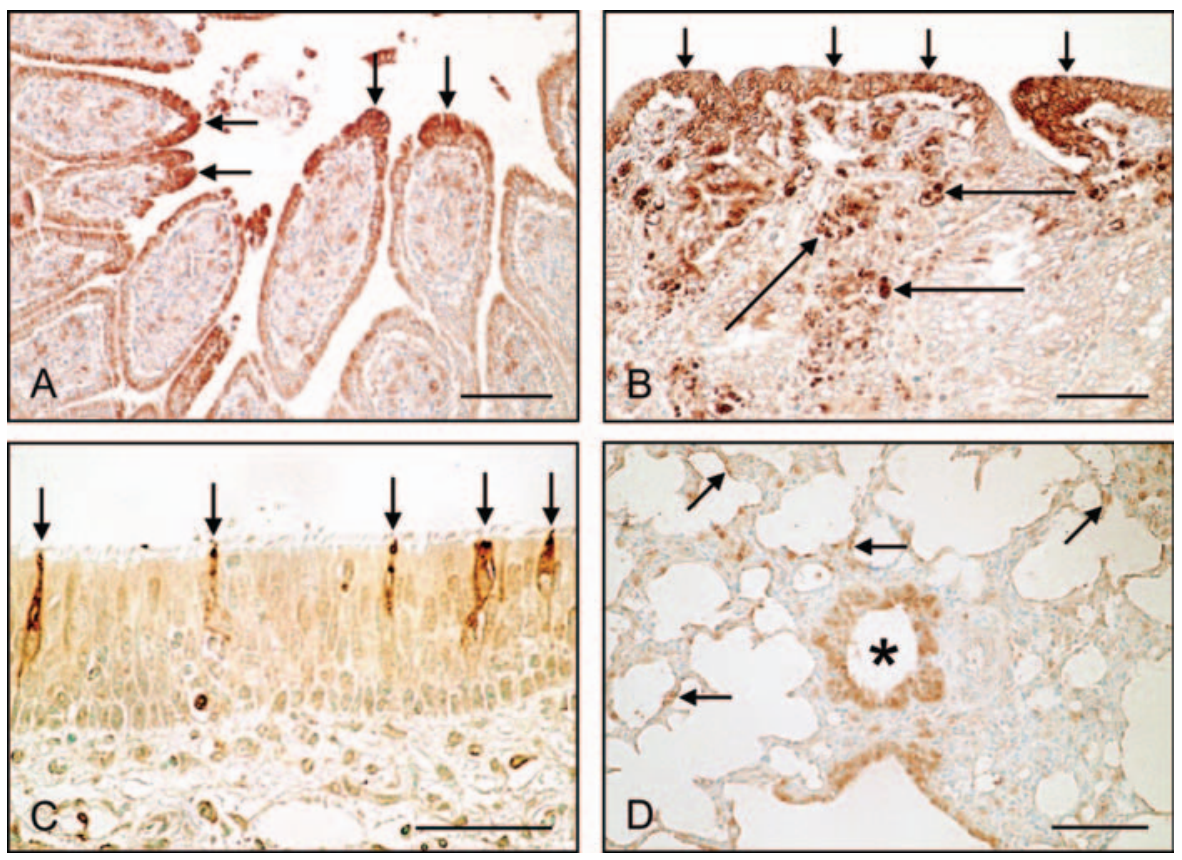

Fig. 3. A Duodenum, colon, nasal turbinates and lungs; BCoV-infected calf. The BCoV antigen in the villous tip epithelial cells (arrows) of the duodenum at PID 1 (A), in the surface epithelium (short arrows) and scattered macrophages (long arrows) of the colon at PID 3 (B), in the epithelium (arrows) of nasal turbinates sampled at PID 3 (C), and in the epithelium of both bronchioles (asterisk) and alveoli (arrows) of the lungs sampled at PID 5 (D) from BCoV-inoculated calves. SAB-PO method, Mayer's hematoxylin counter stain. Bar: A and B, $100 \mu \mathrm{m}$; C, $50 \mu \mathrm{m} ; \mathbf{D}, 200 \mu \mathrm{m}$ 

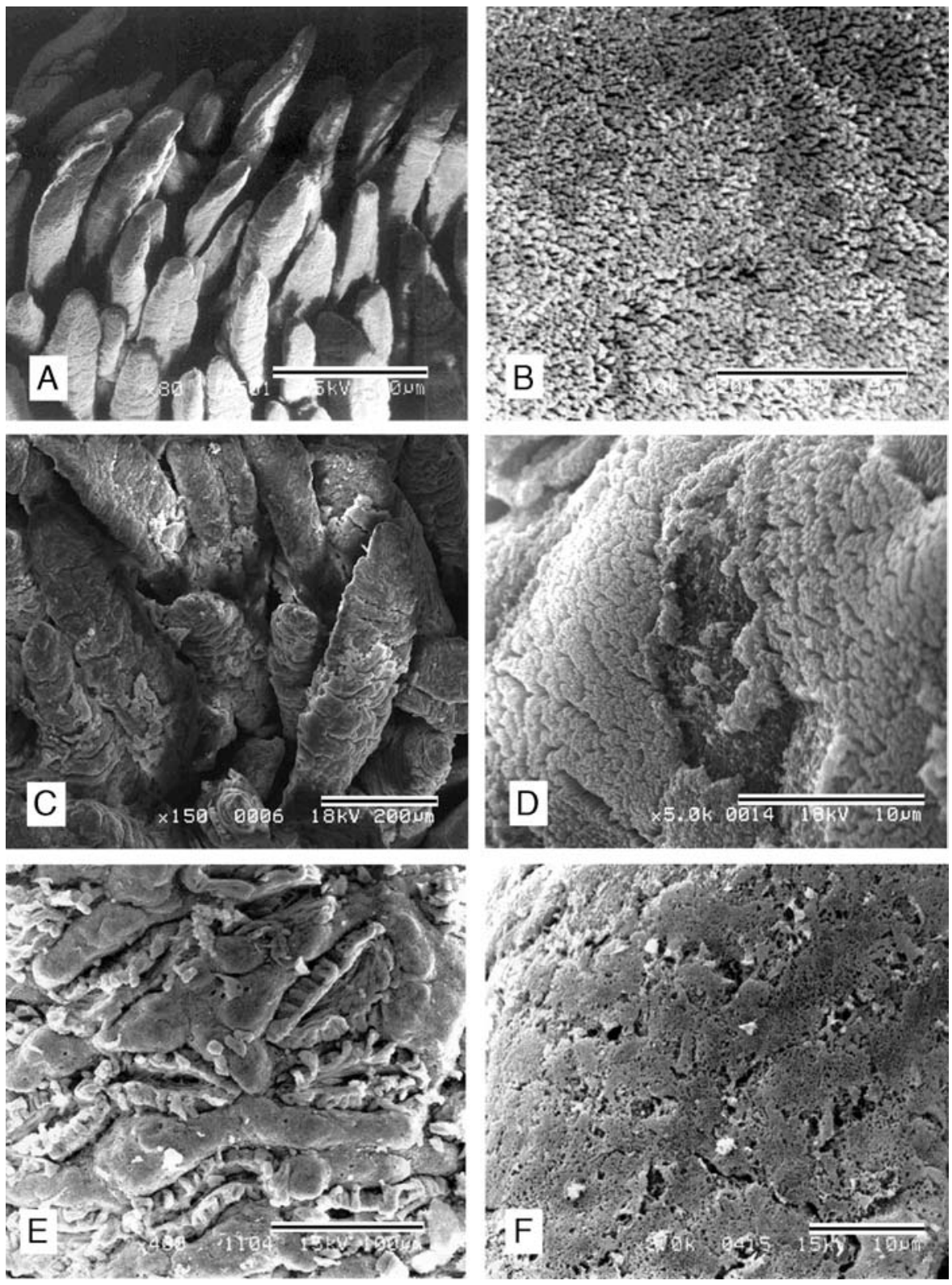

Fig. 4. Scanning electron micrographs of the duodenum; calf. A, B, control animal. A Unaltered long, finger-like villi. B High magnification of $\mathbf{A}$. Note the uniform and densely packed microvillus coat. $\mathbf{C}-\mathbf{F}$, BCoV-infected calf. C Note the multiple desquamations of epithelial lining cells in the villi observed at PID 1. D High magnification of C. Patch exfoliation of microvilli. E Severely shortened and fused villi with the irregular appearance at PID 8. F Magnification of $\mathbf{E}$. Note marked exfoliation of microvilli. Bar: A, $500 \mu \mathrm{m} ; \mathbf{B}, 5 \mu \mathrm{m} ; \mathbf{C}, 200 \mu \mathrm{m} ; \mathbf{D}$ and $\mathbf{F}, 10 \mu \mathrm{m} ; \mathbf{E}, 100 \mu \mathrm{m}$

$B C o V$ antigen distribution in the digestive and respiratory tracts

(i) Intestinal tract. Staining for the $\mathrm{BCoV}$ antigen was confined to the cytoplasm of the tip epithelium of the small intestinal villi, the tip epithelium of the large intestinal glands, and some macrophages scattered in the lamina propria of the large intestine (Fig. 3A and B, Table 2). There was a correlation between $\mathrm{BCoV}$ antigen expression and the histological lesions. At PID
1 , the $\mathrm{BCoV}$ antigen was detected in the epithelium of the duodenum and jejunum, but no histological changes were observed in the jejunum (Table 2). At PID 3, there was a significant increase in the number of antigen-positive cells in the small intestines, which began to appear in some surface epithelia and three to five macrophages of the large intestinal glands (Table 2). At PID 5, the number of antigen-positive cells decreased in the villi of the small intestine but 
increased in the large intestine. The changes in the number of positive cells in the small intestine tended to reflect the desquamation of the epithelial cells in these regions. No positive cells were observed in the small and large intestines of the inactivated KWD3-strain- and mock-inoculated calves (Table 2).

(ii) Respiratory tract. Staining of $\mathrm{BCoV}$ antigens was detected in the cytoplasm of the degenerated and necrotic epithelial cells of the nasal turbinates, trachea and lungs (Fig. 3C and D, Table 3). No BCoV antigen-positive cells were detected in these tissues at PID 1. From PID 3 on, the number increased gradually until PID 8 . There was a consistently higher number of $\mathrm{BCoV}$ antigen-positive cells in the lungs than in the nasal turbinates and trachea. In the lungs, the positive cells included the epithelium of alveoli, bronchi and bronchioles (Fig. 3D). Viral antigens were not detected in the respiratory tract of the inactivated-KWD3-strain- and mock-inoculated calves using the SAB-PO method.
Examination of serum samples from calves inoculated orally with WD-BCoV for BCoV RNA by RT-PCR and nested PCR

RT-PCR detected BCoV RNA in the serum samples from two calves at PID 8 (Table 3). Nested PCR amplified BCoV RNA in the serum samples of all of the inoculated calves at PID 3, 5, and 8 (Table 3). RT-PCR and nested PCR did not detect BCoV RNA in the serum of the inactivated-KWD3-strain- and mock-inoculated calves (Table 3).

\section{Scanning electron microscopy}

The inactivated-KWD3-strain- and mock-inoculated calves exhibited no changes. Long, finger-like small intestinal villi with circular transverse grooves on their surface, and a uniform and densely packed microvillous coat were seen (Fig. 4A and B). The large intestinal epithelium was ridged, with a uniform and densely packed microvillus coat. In the calves inoculated with the KWD3 strain, denuding and shortening of the small intestinal villi and large
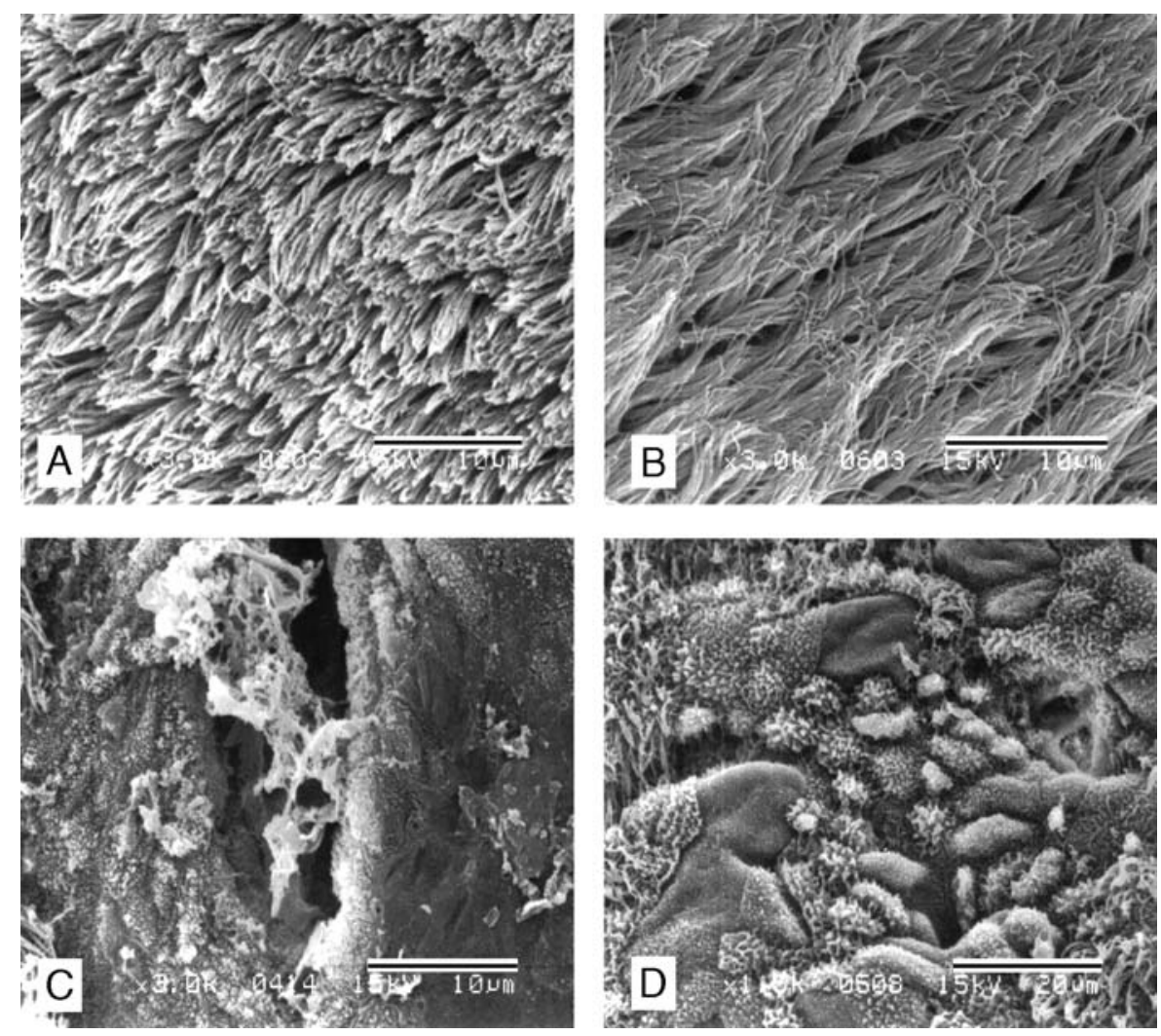

Fig. 5. Scanning electron micrographs of the nasal turbinates and trachea; calf. Normal cilia coat on the surface of the nasal turbinates $\mathbf{A}$ and trachea $\mathbf{B}$. Marked exfoliation of cilia in the nasal turbinates $\mathbf{C}$ and trachea $\mathbf{D}$ at PID 8 from a BCoV-inoculated calf. Bar: A, B and C, $10 \mu \mathrm{m}$; D, $20 \mu \mathrm{m}$ 
intestinal glands were observed, and the microvillus coat was sloughed. At PID 1, in the duodenum patch, exfoliation of the microvilli and epithelial desquamations was observed, with a slight shortening of the villi (Fig. 4C and D). The severity of these morphological changes increased with time. At PID 3 and 5, stunted small intestinal villi and large intestinal glands were fused, and the villi appeared conical or leaf-shaped. At PID 8, some severely stunted villi appeared which exhibited complete exfoliation of microvilli (Fig. 4E and F).

The inactivated-KWD3-strain- and mock-inoculated calves exhibited no respiratory epithelial changes. They showed cilia that completely covered the luminal surface of nasal turbinates and trachea (Fig. 5A and B). However, nasal turbinates and trachea samples from the calves inoculated with the KWD3 strain initially showed patchy exfoliation of the cilia, with complete exfoliation of cilia occurring later (Fig. 5C and D).

\section{Discussion}

It should be noted that the WD-BCoV-induced small and large intestinal lesions were different from those caused by TGEV, which causes only small intestinal lesions [29]. Although $\mathrm{BCoV}$ is believed to replicate in the epithelium of the respiratory tract $[5-7,31]$, the precise regions or cells where $\mathrm{BCoV}$ replicates are still unknown. This study demonstrated that WD-BCoV has tropism for the epithelium of the nasal turbinates, trachea and lungs. Moreover, mild to severe interstitial pneumonia was detected in the BCoV-inoculated calves, suggesting that the WD-BCoV induces interstitial pneumonia. Some pneumotropic viruses, e.g. feline $\mathrm{CoV}$ and canine distemper virus, cause necrosis of the alveolar epithelium, which can lead to compensatory hyperplasia of the type II pneumocytes as well as the infiltration of macrophages and lymphoid cells into the interstitium, resulting in interstitial pneumonia $[10,21]$. Since this study showed that WD-BCoV replicated and damaged the alveolar epithelium, the interstitial pneumonia induced by WD-BCoV might occur through a similar mechanism. However, unlike SARS-CoV, WD-BCoV showed stronger enterotropism than respiratory tro- pism [34]. Moreover, WD-BCoV caused dual lesions in the small and large intestines, even though the lesions in small intestine were more severe than those of the large intestine.

Phylogenetically, the Korean WD-BCoV strains, including the KWD3, are closely related to both respiratory and enteric strains among the other known BCoVs studied in paired comparisons [18]. Moreover, the recently isolated American BCoVs clustered into the same branch with other recently isolated respiratory strains and were distinct from the ancestral enteric Mebus strain [16]. This means that the $\mathrm{BCoV}$ strains might be diverging from an enteric tropism to a dual (respiratory and enteric) tropism over time through intermediates [16, 18]. This study demonstrated that WD-BCoV exhibited both enteric and respiratory tropisms.

Previously, it was not known if $\mathrm{BCoV}$ induced viremia. In this study, WD-BCoV RNA was detected transiently (PID 3) in the serum samples of calves showing that an oral infection leads to viremia. However, evidence for $\mathrm{BCoV}$ replication was first detected in the epithelial cells of the small intestine of a calf at PID 1, but not in the respiratory tract, where evidence of virus replication was first detected at PID 3. The onset and duration of viremia was parallel to nasal virus shedding and the presence of viral antigen and associated lesions in the respiratory tract. These results suggest that the cell-free viremia originated after the intestinal damage, which may have led to the development of respiratory infection and lesions after viremia [2].

Many viruses induce viremia during which the viruses circulate in the blood or blood cells and can spread to the target organs to initiate an infection [34]. However, it is unclear how the $\mathrm{BCoV}$ reaches the circulatory system from intestinal lesions after oral inoculation. One possible path is that cell-free $\mathrm{BCoV}$ penetrates the gut barrier from the luminal side after the destruction of enterocytes in the villi, which would expose the basement membrane, followed by transit into the circulatory system, which can spread to the nasal turbinates, trachea and lungs with replication in the epithelium [2].

Although monocyte-associated $\mathrm{BCoV}$ viremia was not examined in this study, antigen-positive macrophages for $\mathrm{BCoV}$ were detected in the lamina 
propria of the large intestinal glands at PID 3 . Recent studies have shown that a feline $\mathrm{CoV}$ infection leads to the systemic viral spread through monocyte-associated viremia [21]. Therefore, it is possible that $\mathrm{BCoV}$ infections of the respiratory tract may also occur through monocyte-associated viremia, which may originate from the intestines. Further studies will be needed to determine which of these pathways causes viremia.

Another possibility is that a minor aerosol infection might have occurred during oral inoculation or that the virus within the feces of infected animals was inhaled at PID 1, which might have caused virus replication, but with delayed kinetics. Indeed, intravenous $\mathrm{BCoV}$ inoculation results in enteric and nasal virus shedding [Cho and Saif, unpublished data]. This suggests that whatever the cause of the viremia, the viruses in the blood can reach and cause lesions in the epithelial cells in both the intestinal and respiratory tracts. This supports our hypothesis that cell-free viremia from the intestinal tract may causes respiratory tract lesions.

Many viruses that are passaged on a variety of tissue culture cell lines may have amino acid substitutions at the surface of the virus particles, which then may acquire the capacity to enter the cells through alternative receptors [3]. It is likely that cell culture propagation of $\mathrm{BCoV}$ affects the antigenic composition [17], hemagglutination activity [37], and intestinal replication of the virus [20]. Besides, mutations of SARS-CoV were observed in passage 0 [28]. In order to limit the potential effect of cell culture-related nucleotide mutations, this study used a WD-BCoV strain that had been passaged 6 times in HRT-18G cells. However, this cell culture propagation of $\mathrm{WD}-\mathrm{BCoV}$ might result in amino acids changes, particularly the $\mathrm{S}$ protein, leading to a modification of WD-BCoV cell tropism [3].

In this study, the mild villous atrophy caused by necrosis and desquamation of the villi lining cells was confined to the duodenum sampled at PID 1 from a $\mathrm{BCoV}$-infected calf in comparison with normal duodenal villi. Although the present $\mathrm{BCoV}$ caused cytopathic effects to the digestive and respiratory epithelia, its mechanisms are poorly understood, particularly in early infections. The $\mathrm{BCoV}$ was reported to cause a loss of microvilli in the in- testinal epithelium of a $\mathrm{BCoV}$-inoculated calf sacrificed $3 \mathrm{~h}$ after inoculation [26], resulting in diarrhea in experimentally inoculated and naturally infected calves. However, the present histological, IHC and SEM studies do not allow a rigorous reconstruction of the sequence of the loss of microvilli during the initial WD-BCoV infections of the digestive tract because transmission electron and immuno-electron microscopic studies were not performed. These analyses would be particularly useful with the specimens sampled $3 \mathrm{~h}$ after inoculation, which presumably would have provided more detailed data on their multiplication and fine structural changes. It has been reported [22] that some mouse hepatitis coronavirus (MHV) strains rapidly reduce the level of actin mRNA, whose protein interacts with microtubules. As a consequence, the virus-induced impairment of the actin filaments interacting with microtubules might lose their connection with the plasma membrane covering the microvillus or anchored cilia, resulting in the loss of microvilli or cilia [4]. In addition, TGEV and some MHV strains induce apoptosis in some cell types $[1,12]$. Therefore, it is possible that the loss of microvilli and cilia induced by WD-BCoV in the digestive and respiratory tracts might occur through a similar mechanisms such as a combination of MHV- or TGEV-induced effects [23].

Our results suggest that the intestinal lesions caused by WD-BCoV spread from the small intestine to the large intestine. With time, the morphological changes in the duodenum became increasingly severe, which led to villous atrophy and fusion. Eventually, the duodenum showed severe villous atrophy, and the other parts of the intestines including the jejunum, ileum, cecum, colon and rectum exhibited moderate to marked mucosal lesions at PID 8. These changes in the lesions are similar to those of calves inoculated with the CD-BCoV strain in which the lesions were first observed in the small intestine and then extended to the large intestine $[13,25,26,31]$.

In the present study, WD-BCoV induced pathological changes in the respiratory tract. These lesions may play as a predisposing factor for secondary bacterial pathogens such as Mannheimia spp. [10, 38]. In addition, a concurrent infection can exacer- 
bate the clinical signs of respiratory tract disease [11]. Considering the acknowledged roles of other viruses in the bovine respiratory disease complex $[11,38]$, it is possible that WD-BCoV may also act synergistically with other infectious agents and stressors to cause pneumonia in calves [38].

\section{Acknowledgements}

This work was supported by Chonnam National University and the Regional Technology Innovation Program of the Ministry of Commerce, Industry and Energy (MOCIE), Republic of Korea. The authors acknowledge a graduate fellowship provided by the Korean Ministry of Education and Human Resources Development through the Brain Korea 21 project.

\section{References}

1. An S, Chen CJ, Yu X, Leibowitz JL, Makino S (1999) Induction of apoptosis in murine coronavirus-infected cultured cells and demonstration of $\mathrm{E}$ protein as an apoptosis inducer. J Virol 73: 7853-7859

2. Azevedo MS, Yuan L, Jeong KI, Gonzalez A, Nguyen TV, Pouly S, Gochnuer M, Zhang W, Azevedo A, Saif LJ (2005) Viremia and nasal and rectal shedding of rotavirus in gnotobiotic pigs inoculated with Wa human rotavirus. J Virol 79: 5428-5436

3. Baranowski E, Ruiz-Jarabo CM, Pariente N, Verdaguer N, Domingo E (2003) Evolution of cell recognition by viruses: a source of biological novelty with medical implications. Adv Virus Res 62: 19-111

4. Cheville NF (1994) Interpretation of acute cell injury: degeneration. In: Cheville NF (ed) Ultrastructural pathology: an introduction to interpretation. Iowa State University Press, Iowa, pp 51-79

5. Cho KO, Hoet AE, Loerch SC, Wittum TE, Saif LJ (2001) Evaluation of concurrent shedding of bovine coronavirus via the respiratory tract and enteric route in feedlot cattle. Am J Vet Rec 62: 1436-1441

6. Cho KO, Hasoksuz M, Nielsen PR, Chang KO, Lathrop S, Saif LJ (2001) Cross-protection studies between respiratory and calf diarrhea and winter dysentery coronavirus strains in calves and RT-PCR and nested PCR for their detection. Arch Virol 146: 2401-2419

7. Cho KO, Halbur PG, Bruna JD, Sorden SD, Yoon KJ, Janke BH, Chang KO, Saif LJ (2000) Detection and isolation of coronavirus from feces of three herds of feedlot cattle during outbreaks of winter dysentery-like disease. J Am Vet Med Assoc 217: 1191-1194

8. Cho KO, Ohashi K, Onuma M (1999) Electron microscopic and immunohistochemical localization of Marek's disease (MD) herpesvirus particles in MD skin lymphomas. Vet Pathol 36: 314-320
9. Chouljenko VN, Kousoulas KG, Lin X, Storz J (1998) Nucleotide and predicted amino acid sequences of all genes encoded by the $3^{\prime}$ genomic portion $(9.5 \mathrm{~kb})$ of respiratory bovine coronaviruses and comparisons among respiratory and enteric coronaviruses. Virus Genes 17: 33-42

10. Dungworth DL (1993) The respiratory system. In: Jubb KVF, Kennedy PC, Palmer N (eds) Pathology of domestic animals. Academic Press, San Diego, pp 539-699

11. Dyer RM (1981) The bovine respiratory disease complex: infectious agents. Compend Contin Educ Pract Vet 3: S374-S382

12. Eleouet JF, Chilmonczyk S, Besnardeau L, Laude H (1998) Transmissible gastroenteritis coronavirus indues programmed cell death in infected cells through a caspase-dependent pathway. J Virol 72: 4918-4924

13. El-Kanawati ZRH, Tsunemitsu H, Smith DR, Saif LJ (1996) Infection and cross-protection studies of winter dysentery and calf diarrhea bovine coronavirus strains in colostrum-deprived and gnotobiotic calves. Am J Vet Res 57: 48-53

14. Flynn WT, Saif LJ, Moorhead PG (1988) Pathogenesis of porcine enteric calicivirus in four-day-old gnotobiotic piglets. Am J Vet Res 49: 819-825

15. Gallagher TM, Buchmeier MJ (2001) Coronavirus spike proteins in viral entry and pathogenesis. Virology 279: 371-374

16. Hasoksuz M, Sreevatsan S, Cho KO, Hoet AE, Saif LJ (2002) Molecular analysis of the S1 subunit of the spike glycoprotein of respiratory and enteric bovine coronavirus isolates. Virus Res 84: 101-109

17. Hussain KA, Storz J, Kousoulas KG (1991) Comparison of bovine coronavirus (BCV) antigens: monoclonal antibodies to the spike glycoprotein distinguish between vaccine and wild-type strains. Virology 183 : $442-445$

18. Jeong JH, Kim GY, Yoon SS, Park SJ, Kim YJ, Sung CM, Shin SS, Lee BJ, Kang MI, Park NY, Koh HB, Cho KO (2005) Molecular analysis of S gene of spike glycoprotein of winter dysentery bovine coronavirus circulated in Korea during 2002-2003. Virus Res 108: 207-212

19. Jeong JH, Kim GY, Yoon SS, Park SJ, Kim YJ, Sung CM, Shin SS, Koh HB, Lee BJ, Lee CY, Kang MI, Kim HJ, Park NY, Cho KO (2005) Detection and isolation of winter dysentery bovine coronavirus circulated in Korea during 2002-2004. J Vet Med Sci 67: 187-189

20. Kapil S, Ttrent AM, Goyal SM (1990) Excretion and persistence of bovine coronavirus in neonatal calves. Arch Virol 115: 127-132

21. Kipar A, May H, Menger S, Weber M, Leukert W, Reinacher M (2005) Morphologic features and development of granulomatous vasculitis in feline infectious peritonitis. Vet Pathol 42: 321-330 
22. Kyuwa S, Cohen M, Nelson G, Tahara SM, Stohlman SA (1994) Modulation of cellular macromolecular synthesis by coronavirus: implication for pathogenesis. J Virol 68: 6815-6819

23. Lai MMC, Holmes KV (2001) Coronaviridae: the viruses and their replication. In: Fields BN, Knipe DM, Howley PM (eds) Fields virology. Lippincott-Raven, Philadephia, pp 1163-1185

24. Lathrop SL, Wittum TE, Loerch SC, Perino LJ, Saif LJ (2000) Antibody titers against bovine coronavirus and shedding of the virus via the respiratory tract in feedlot cattle. Am J Vet Res 61: 1057-1061

25. Mebus CA (1978) Pathogenesis of coronaviral infection in calves. J Am Vet Med Assoc 173: 631-632

26. Mebus CA, Stair EL, Rhodes MB, Twiehaus MJ (1973) Pathology of neonatal calf diarrhea induced by a coronavirus-like agent. Vet Pathol 10: 45-64

27. Park SJ, Jeong C, Yoon SS, Choy HE, Saif LJ, Park SH, Kim YJ, Jeong JH, Park SI, Kim HH, Lee BJ, Cho HS, Kim SK, Kang MI, Cho KO (2006) Detection and characterization of bovine coronaviruses in fecal specimens of adult cattle with diarrhea during the warmer seasons. J Clin Microbiol 44: 3178-3188

28. Poon LL, Leung CS, Chan KH, Yuen KY, Guan Y, Peiris JS (2005) Recurrent mutations associated with isolation and passage of SARS coronavirus in cells from non-human primates. J Med Virol 76: 435-440

29. Saif LJ, Wesley RD (1999) Transmissible gastroenteritis and porcine respiratory coronavirus. In: Straw BE, D’Allaire S, Mengeling WL, Taylor DI (eds) Diseases of swine. Iowa State University Press, Ames, Iowa, pp 295-325

30. Saif LJ (1990) A review of evidence implicating bovine coronavirus in the etiology of winter dysentery in cows: an enigma resolved? Cornell Vet 80: 303-311

31. Saif LJ, Redman DR, Moorhead PD, Theil KW (1986) Experimentally induced coronavirus infections in calves: viral replication in the respiratory and intestinal tracts. Am J Vet Res 47: 1426-1432

32. Schultze B, Herrler G (1993) Recognition of N-acetyl9-O-acetylneuraminic acid by bovine coronavirus and hemagglutinating encephalomyelitis virus. Adv Exp Med Biol 342: 299-304

33. Schultze B, Gross HJ, Brossmer R, Herrler G (1991) The $\mathrm{S}$ protein of bovine coronavirus is a hemagglutinin recognizing 9-O-acetylated sialic acid as a receptor determinant. J Virol 65: 6232-6237

34. Shi X, Gong E, Gao D, Zhang B, Zheng J, Gao Z, Zhong Y, Zou W, Wu B, Fang W, Liao S, Wang S, Xie Z, Lu M, Hou L, Zhong H, Shao B, Li N, Liu C, Pei F, Yang J, Wang Y, Han Z, Shi X, Zhang Q, You J, Zhu X (2005) Severe acute respiratory syndrome associated coronavirus is detected in intestinal tissues of fatal cases. Am J Gastroenterol 100: 169-176

35. Silim A, Elazhary MA (1983) Detection of infectious rhinotracheitis and bovine viral diarrhea virus in the nasal epithelial cells by the direct immunofluorescence technique. Can J Com Med 47: 18-22

36. Smith DR, Tsunemitsu H, Heckert RA, Saif LJ (1996) Evaluation of two antigen-capture ELISAs using polyclonal or monoclonal antibodies for the detection of bovine coronavirus. J Vet Diagn Invest 8: 99-105

37. Storz J, Zhang XM, Rott R (1992) Comparison of hemagglutinating, receptor destroying, and acetylesterase activities of avirulent and virulent bovine coronavirus strains. Arch Virol 125: 193-204

38. Straub OC (1995) Viral respiratory infections of cattle. Bovine Pract 29: 66-70

39. Traven M, Naslund K, Linde N, Linde B, Silvan A, Fossum C, Hedlund KO, Sarsson B (2001) Experimental reproduction of winter dysentery in lactating cows using BCV - comparison with BCV infection in milkfed calves. Vet Microbiol 81: 127-151

40. Tsunemitsu H, Saif LJ (1995) Antigenic and biological comparisons of bovine coronaviruses derived from neonatal calf diarrhea and winter dysentery of adult cattle. Arch Virol 140: 1303-1311

41. Welch SK, Saif LJ (1988) Monoclonal antibodies to a virulent strain of transmissible gastroenteritis virus: comparison of reactivity with virulent and attenuated virus. Arch Virol 101: 221-235 